\title{
Management of Allergic Diseases During COVID-19 Outbreak
}

\author{
Adriana Izquierdo-Domínguez ${ }^{1,2,3}$ (D) María Jesús Rojas-Lechuga ${ }^{4,5,6} \cdot$ Isam Alobid M $^{2,4,5,6}$ \\ Accepted: 13 January 2021 / Published online: 9 February 2021 \\ (C) The Author(s), under exclusive licence to Springer Science+Business Media, LLC part of Springer Nature 2021
}

\begin{abstract}
Purpose of Review The coronavirus disease 2019 (COVID-19) has challenged healthcare system capacities and safety for health care workers, reshaping doctor-patient interaction favoring e-Health or telemedicine. The pandemic situation may make difficult to prioritize patients with allergies diseases (AD), face-to-face evaluation, and moreover concern about the possible COVID-19 diagnosis, since COVID-19 shared many symptoms in common with AD. Being COVID-19 a novel disease, everyone is susceptible; there are some advances on vaccine and specific treatment. We evaluate existing literature on allergic diseases (AD): allergic rhinitis, asthma, food allergy, drug allergy, and skin allergy, and potential underlying mechanisms for any interrelationship between AD and COVID-19.

Recent Findings There is inconclusive and controversial evidence of the association between AD and the risk of adverse clinical outcomes of COVID-19. AD patients should minimize hospital and face-to-face visits, and those who have used biologics and allergen immunotherapy should continue the treatment. It is essential to wear personal protective equipment for the protection of health care workers.

Summary Social distancing, rational use of facemasks, eye protection, and hand disinfection for health care workers and patients deserve further attention and promotion. Teleconsultation during COVID-19 times for AD patients is very encouraging and telemedicine platform can provide a reliable service in patient care.
\end{abstract}

Keywords Allergic diseases · Coronavirus disease 2019 (COVID-19) · Food allergy · Drug allergy · Skin allergy · Allergic asthma $\cdot$ Allergic rhinitis $\cdot$ Pandemic

\section{Introduction}

The coronavirus disease 2019 (COVID-19) caused by the severe acute respiratory syndrome coronavirus 2 (SARS-CoV2) infection shared many symptoms in common with allergic

This article is part of the Topical Collection on Rhinosinusitis

Adriana Izquierdo-Domínguez

adrianaeizquierdo@hotmail.com

1 Department of Allergology, Consorci Sanitari de Terrassa, Barcelona, Spain

2 Unidad Alergo-Rino, Centro Médico Teknon, Barcelona, Spain

3 Department of Allergy, Clínica Diagonal, Barcelona, Spain

4 Rhinology Unit \& Smell Clinic, Department of Otorhinolaryngology, Hospital Clinic Barcelona, Universitat de Barcelona, Barcelona, Spain

5 Clinical and Experimental Respiratory Immunoallergy, IDIBAPS, Barcelona, Spain

6 CIBER of Respiratory Diseases (CIBERES), Madrid, Spain diseases (AD), such as nasal congestion, cough, shortness of breath, and olfactory and taste dysfunction. That overlapping of respiratory symptoms can make difficult physician's management of $\mathrm{AD}$, can be confusing for patients, and may delay seeking for medical help $[1,2]$.

The COVID-19 outbreak challenged healthcare systems capacities, and reshaped doctor-patient interaction, favoring remote video or phone consultation in the cases that allow it [3]. It created many issues around the optimal and safest way to treat complex patients with $\mathrm{AD}$.

In the case of need for a face-to-face consultation or performed complementary tests, carry out surveys regarding symptoms suggestive of COVID-19 or possible risk's contacts to our patients who will come for present visits is highly recommended. The general measures of hand hygiene, distancing, use of a mask, correct ventilation, and cleaning of the facilities must be respected. It is essential to take precautionary measures by health professionals with appropriate personal protective equipment (PPE) with special attention on aerosolizing procedures, follow local recommendations, and to assess the need for examinations and treatments for our patients on a case-by-case basis (Table 1) $[4,5]$. 
This review provides a comprehensive overview of the management of AD during the COVID-19 pandemic situation.

\section{Allergic rhinitis}

Allergic rhinitis (AR) is defined as an inflammation of the nasal mucosa by an immunoglobulin E (IgE)-mediated response to an extrinsic protein [6]. AR is characterized by sneezing, nasal congestion, nasal itching, and rhinorrhea; other symptoms include palate itching, postnasal drip, cough [7], and olfactory dysfunction [8]. It is frequently associated to allergic rhino-conjunctivitis (tearing, itching, and redness of the eyes), asthma (15-38\% of patients with AR) [7], and atopic dermatitis [9]. AR could be classified as intermittent or persistent, and by severity as mild, moderate, or severe according to the ARIA guideline. The diagnosis must be confirmed by an allergy skin test (immediate hypersensitivity testing), and other complementary tests could be considered. $\mathrm{AR}$ is one of the most common $\mathrm{AD}$ and affects 10 to $40 \%$ of worldwide population [10]. Although is not a life-threating condition, it can significantly impair quality of life [7].

The AR treatment could be composed by 3 major categories: (a) environmental control measures and allergen avoidance [11], (b) pharmacological treatment as a single or a combination of oral or intranasal $\mathrm{H}_{1}$-antihistamine, intranasal corticosteroids, MP-AzeFlu (fluticasone plus azelastine intranasal formulation), or leukotriene receptor antagonist, and (c) specific allergen immunotherapy (AIT) [12].

Until the date, there is a limited knowledge on the differences in the COVID-19 severity of the illness in allergic compared to non-allergic patients. Yang et al. in a Korean cohort study, showed AR was associated with an increased likelihood of SARS-CoV-2 test positivity and worth clinical outcomes (i.e., death, ICU admission, and invasive ventilation) and longer hospital stay [13]. Opposite to the above, Chhiba et al. observed that rhinosinusitis was associated with lower risk of hospitalization, and AR also showed a trend toward lower hospitalization although not statistically significant in
COVID-19 patients with or without asthma [2]. More studies are needed to clarify the relationship between COVID-19 severity and AR.

General recommendations for AR patients during COVID19 outbreak (Table 2) [3, 5, 14]:

1. Face-to-face evaluation and management of AR patients can be postponed or can be implemented by remote health care tools for first visits or monitored patients.

2. Advise patients to continue their AR treatments, including intranasal corticosteroids, second-generation $\mathrm{H}_{1}$-antihistamines, or MP-AzeFlu [15].

3. Patients can be instructed on allergen avoidance measures and treatment modalities.

4. Considering allergen immunotherapy, it is suggested to continue with the treatment in case of non-infected patients or recovered from COVID-19 infection, and suspended in case of positive or suspected SARS-CoV-2 infection [16].

5. Follow infection control recommendations and PPE if aerosol-generating procedures are needed.

\section{Allergic Asthma}

Asthma is a chronic disease usually characterized by chronic airway inflammation. It presents with acute exacerbations being a frequent cause of emergency room visits and/or hospitalizations $\left[17^{\circ}\right]$. The diagnosis is clinical with a history of fluctuating respiratory symptoms such as shortness of breath, wheeze, chest tightness, and cough associated to a variable expiratory airflow limitation. The diagnosis should be confirmed with a spirometry/PEF with reversibility test. Asthma affects an estimated 300 million individuals worldwide although there are many geographical differences with prevalence between 1 and $18 \%$ of the population [18]. It has a significant impact on health-related quality of life (QoL) and familiar disruption, especially in pediatric asthma, and is associated with substantial healthcare and productivity costs [18].

Table 1 General recommendations in patients with allergic diseases during COVID-19 pandemic situation

Prioritizing care of ambulatory visits

Adjust to the local recommendations depends on the threat levels. Triaging allergy patients by diagnoses, and severity is recommended considering short-term rationing services (6 months)

Face-to-face visit

Telehealth

Prevention measures for health care workers
Limit face-to-face patient interactions where possible. Conduct correctly patients with COVID-19 symptoms, and those with AD decompensations

Well-controlled allergic diseases are appropriately for telehealth visits during face-to-face consultation are postposed. Also monitor treatments, and first visits.

Use of personal protective equipment including standard, contact, and airborne precautions, including gown, gloves, goggles, and an N95 mask or powered air-purifying respirator. 


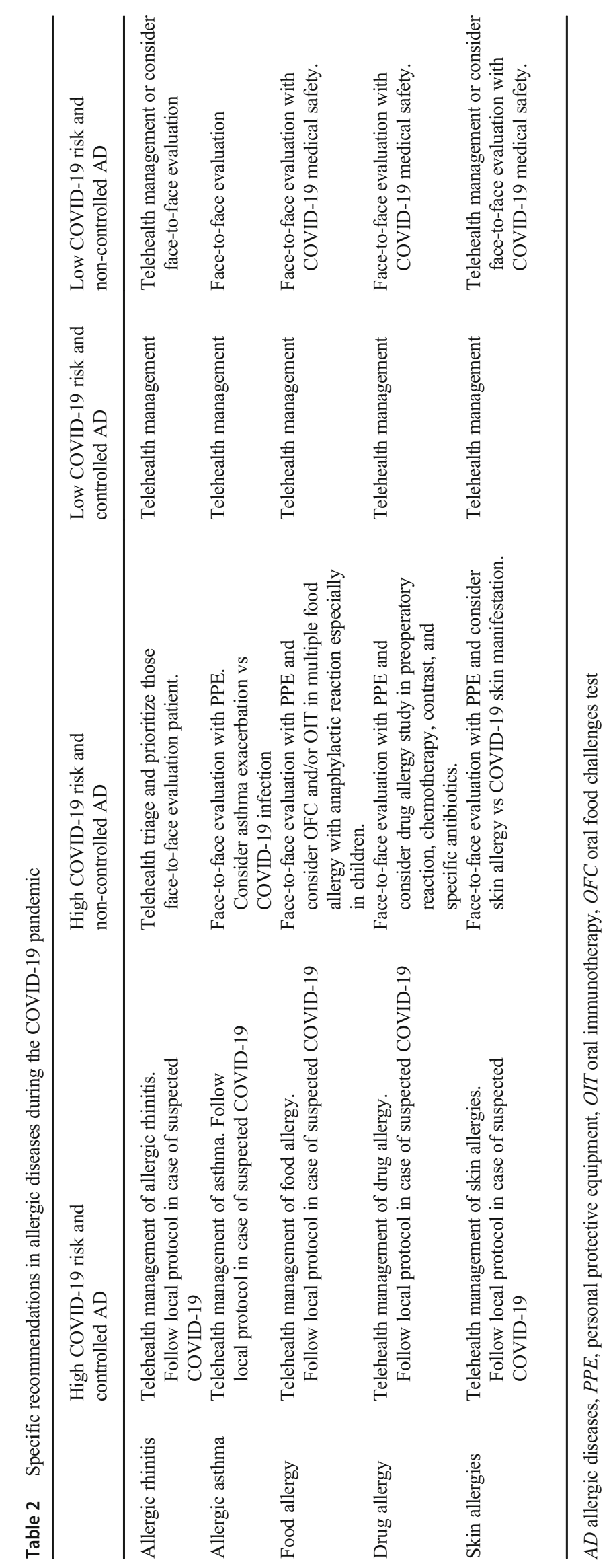


Asthma is a heterogeneous disease with different clinical phenotypes proposed, the most easily recognized is allergic asthma, which usually debut in childhood, and is associated with a past and/or family history of $\mathrm{AD}$ such as eczema, AR, and food or drug allergy. It usually presents eosinophilic airway inflammation and responds well to inhaled corticosteroid treatment $[18,19]$.

Respiratory viruses are well-known triggers of asthma exacerbations [1]. Until the date, there is no data to demonstrate a specific increased risk for asthma exacerbation caused by SARS-CoV-2 infection in patients with asthma. However, this association could evolve [3]. Medical societies and organizations have warned about the difficulty in distinguishing between symptoms of COVID-19 and those of severe asthma exacerbation $\left[17^{\circ}\right]$. Abrams et al. showed that fever may help differentiate between asthma exacerbations and COVID-19 in pediatric and adult populations, but caution should still be taken as fever may be present in other virus-triggered asthma exacerbations [20]. Other symptoms that are better described in adults that are more likely to suggest COVID-19 infection include myalgia, headache, pharyngitis, rhinorrhea, loss of smell and/or taste [21], diarrhea, nausea, and vomiting [20].

Although there is limited data about the impact of many underlying medical conditions, the Centers for Disease Control (CDC) classifies patients with moderate to severe asthma as conditions that might be at an increased risk for severe COVID-19 illness [22].

Concerning physiopathology, SARS-CoV-2 binds to angiotensin-converting enzyme 2 (ACE2) receptor in respiratory mucosa (nasal and lower airway epithelial cells) by receptor respiratory domain S1 with the help of the cellular transmembrane protease serine S1 member 2 (TMPRSS2). The binding of the SARS-CoV-2 to ACE2 increases the expression of ACE2 with the consequent damage of the alveolar cells. Until the date, still remains unclear if SARS-CoV-2 may be a trigger for asthma exacerbation. Hypotheses favoring that relationship propose that like other respiratory viruses, COVID-19 induces an attenuated interferon (IFN)-I and IFN-III response, associated with high levels of proinflammatory cytokines and their receptors, resultant in an unopposed $\mathrm{T}_{\mathrm{H}} 2$ response that can possibly contribute to asthma exacerbation, also patients with AA have higher nasal levels of TMPRSS2, and it is proposed as a feasible risk factor for COVID-19 severity. On the other side, patients with AA ( $\mathrm{T}_{\mathrm{H}}$ 2-high endotype) have fewer receptors for SARS-CoV-2 entry, associated with downregulation of ACE2 with environmental allergens exposure, and may protect them from COVID-19 [1].

In accordance with the abovementioned, in literature, a retrospective study conducted in Chicago from Chhiba et al. observed no significant difference in risk of hospitalization or mortality due to COVID-19 in patients with or without asthma [2]. In contrast, on April 8, 2020, the Morbidity and Mortality
Weekly Report shows that $27 \%$ of patients hospitalized for COVID-19 between 18 and 49 years old had asthma [1]. Considering a $10 \%$ prevalence in the USA confers asthma an increased risk of hospitalization. Williamson et al. found that asthma was associated with an increased risk of inhospital death from COVID-19 adjusted by age and sex [23]. More evidence is needed to clarify the impact of asthma in COVID-19 patients or vice versa.

General recommendations for asthma patients during COVID-19 outbreak (Table 2) [3, 17•, 18, 24]:

1. Promote e-health and telemedicine in those patients who consider appropriate.

2. Advise patients to continue taking their prescribed asthma medications (including oral or inhaled corticosteroid and add-on biologic therapy in case of severe asthma) with consideration given to training to self-administer, and remote monitoring.

3. Avoid known triggers including smoking and secondhand smoke exposure.

4. Make sure that all patients have a written asthma action plan, specifying how to increase their reliever and controller medications and when to seek medical help.

5. Advise patients to wash their hands and clean equipment regularly. Avoid nebulizers due to risk of aerosolizing. Consider measure peak flow diaries and favor personaluse peak flow meter.

6. Only carrying out bronchoscopy and pulmonary function tests for urgent cases where results will have a direct impact on patient care (as these tests may spread COVID19).

7. Follow infection control recommendations and PPE if aerosol-generating procedures are needed.

\section{Food allergy}

Food allergy (FA) can result in considerable morbidity and impact negatively on costly in terms of medical care and QoL. FA can result in life-threatening anaphylaxis. The prevalence of FA was generally higher in children than in adults. The management of optimal and organized strategies are used to minimize future allergic reactions and improve the QoL [25].

Due to the virus that will circulate for the next months, the management of FA was initially deprioritized and adapted to current condition (Fig. 1). In the setting of ongoing deferment of many FA services, alternative management strategies exist to provide care. Patient concerns and priorities must be incorporated into a model of shared decision making regarding alternatives to traditional care and management include (a) food-allergy prevention in infants, (b) anaphylaxis treatment, (c) provision of oral food challenges (OFCs), and (d) oral 
immunotherapy (OIT). The constraints of the pandemic that have been forced upon the specialty provide an opportunity for FA management to evolve.

The complexity and high risk of severe allergic reactions due to FA, especially in patients with multiples FA and in children, so that making them priority patients in daily clinical practice. In FA study in children, their mothers reported high anxiety and poor healthrelated QoL. Yet, qualitatively, day-to-day FA management was better during the pandemic, due to daily monitoring on the children [26].

Leveraging virtual care and incorporating more allowances for parents to facilitate FA management are strategies that can improve outcomes while balancing risk. Patient concerns and priorities must be considered into a model of shared decision making regarding alternatives to actual management.

General recommendations for food allergy patients during COVID-19 outbreak [16, 27]:

1. Promote telehealth and incorporating more allowances for parents to facilitate FA management are strategies that can improve outcomes while balancing risk.

2. Follow local recommendations, and prioritize when possible a face-to-face consultation in patients with a high risk of severe reaction and multiple FA.

3. In patients diagnosed with COVID-19 or cases with suspected SARS-CoV-2 infection, OIT dosing should continue as indicated in the dosing plan and in coordination with the treating physician.

4. OIT can be continued in non-infected patients and those who have recovered from COVID-19.

5. In areas with high level of SARS-CoV-2 community transmission, visits to the allergy clinic for OIT updosing should be postponed.

\section{Drug Allergy}

Drug hypersensitivity reactions are a concern for physicians and patients. They have unique clinical features, specific diagnostic methods, and their own implications for causative drugs. Drug allergies (DA) affect more than $7 \%$ of the worldwide population; the skin is the most frequently involved organ, with a broad spectrum of reaction types with different morphologies, chronologies, and mechanisms [28].

Life-threatening hypersensitivity reactions are more likely to occur in patients with a history of allergy, atopy, or asthma. Hence, in a patient who presented with a history of multiple DA, an allergology assessment should be performed prior.

Drug provocation tests are not recommended during the COVID-19 pandemic, because reactions can occur during the tests, including the generation and spreading of viruscontaining aerosols. However, they may be considered after

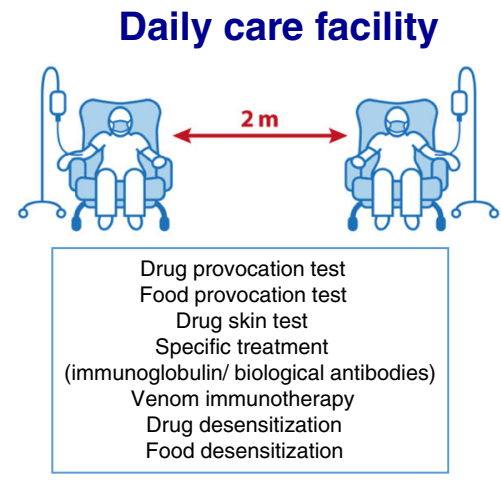

Hospitalization
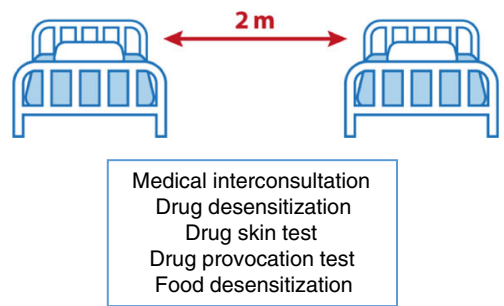

\section{Physician`s office}

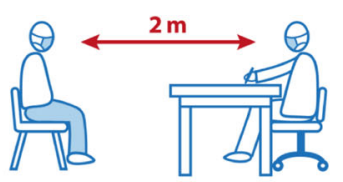

\section{Physician examination}
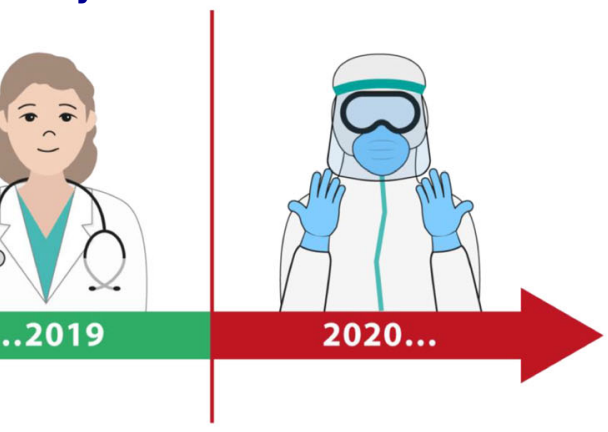

Diagnostic test room

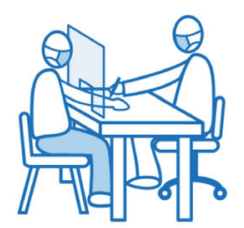

Spirometry/Oscilometry Nasal/bronchial challenge test Cardiopulmonary exercise testing Bronchial nitric oxide Induced sputum Nasal sampling Nasal nitric oxide Smell test/ Chemical gustometry Allergen specific immunotherapy Skin prick test/ Patch test Acoustic rhinometry

Anterior rhinomanometry Peak nasal inspiratory flow

Fig. 1 Flow chart of how COVID-19 changes our daily allergology practices and interventions 
careful risk-benefit assessment in cases of urgent need, such as chemotherapy in cancer patients, radiocontrast media in subjects needing urgent procedures, and DA reaction during the perioperative time. Given that DAs are causes of catastrophic events occurring in the perioperative period, they are of major concern to anesthesiologists [29]. Also, in case of specific antibiotics and if no effective alternative drug is available, it may be considered the preferent allergology study $\left[5^{\circ}\right]$.

Additionally, DA has to be considered as a differential diagnosis against skin lesions due to COVID-19 that could be related to thrombovascular events (i.e., petechiae, acro-ischemia, dry gangrene) or to typical viral infections (i.e., erythematous rash, urticaria, maculopapular exanthema) [30], being a difficult distinction during the acute phase. Diagnosis relies mostly on clinical observations. In that regard, an accurate chronology of the reaction and the drug exposure timeline are very informative and important in the allergology study [28]. Laboratory and histopathological findings may also help.

Despite a great effort to flatten the curve of infections, the spread of this global pandemic continues to expand. As more people are exposed to different and multiple treatments to try to control COVID-19, the risk of new DA reactions from these drugs increases.

The immunomodulatory drugs, including azithromycin, hydroxychloroquine/chloroquine, and IFNs, are the ones most frequently involved in hypersensitivity reactions. Most reactions are non-immediate, and further studies are required to clarify whether this will probably be associated with a rise in the number of drug-related adverse effects, some of which have an immunological basis [30].

Dordal et al. summarized the current knowledge regarding mainly the immunological adverse drug reactions related to the drugs used for COVID-19, in order to identify them early and address their management in a comprehensive manner and provide the allergology study [31•].

General recommendations for DA patients during COVID19 outbreak and prioritize allergology drug study [30]:

1. Chemotherapy in cancer patients.

2. Radiocontrast media in subjects needing urgent procedures.

3. Drug allergy reaction during the perioperative period.

4. Antibiotics if no effective alternative drugs are available.

5. Patients with suspected COVID-19 and skin reactions.

6. COVID-19-positive patients who have received multiple immunomodulatory drugs.

\section{Skin Allergy}

Allergic contact dermatitis and atopic eczema are dermatologic diseases that share work with allergists. COVID-19 patients had a broad spectrum of cutaneous lesions that can overlap with skin allergy diseases (SAD), that range from an unspecific macular erythematous rash, urticarial lesions, chickenpox-like vesicles, and acroischemic lesions [32]. They can result from local inflammation due to circulating immune complexes or from systemic manifestations leading to vasculitis and thrombosis [33]. Furthermore, COVID-19 patients are also at increased risk of drug hypersensitivity lesions [30].

There is no evidence that patients with barrier defects such as atopic eczema have a higher risk for SARS-CoV-2 infection or skin complications during COVID-19. However, patients with atopic dermatitis are often on systemic immunosuppressants and should be monitored closely. Optimal topical treatment regime should also be encouraged in all patients [34].

In the case of urgently indicated systemic therapy for severe atopic dermatitis, consideration should be given to therapy with either biologicals, classic immunosuppressants, or systemic glucocorticoids, although systemic glucocorticoids are not recommended due to their broad immunosuppressive effect. Severe COVID-19 courses have been reported in two patients with atopic dermatitis treated with dupilumab [35].

General recommendations for skin allergy patients during COVID-19 outbreak [36]:

1. Effective skin-care after hand hygiene is therefore essential to prevent barrier disruption and sensitization events.

2. Emollients containing hyaluronic acid, Vitamin E, ceramide or urea are recommended.

\section{Specific allergic treatments: Immunotherapy and biological monoclonal antibodies}

Since the beginning of the COVID-19 pandemic, the treatment of patients with atopy and $\mathrm{AD}$ has faced major challenges.

\section{Allergen-specific immunotherapy}

It is the only causal therapy with a proven long-term benefit in allergic airway diseases, such as AA or AR. AIT may prevent asthma onset in patients with AR [37].

Specifically, in the cases of hymenoptera venom, allergy is a potentially life-threatening allergic reaction owing to honeybee, vespids, or ant sting. Systemic-allergic sting reactions have been reported in up to $7.5 \%$ of adults and up to $3.4 \%$ of children. They can be mild and restricted to the skin or moderate to severe with a risk of life-threatening anaphylaxis. Patients should carry an emergency kit containing an adrenaline autoinjector, $\mathrm{H}_{1}$-antihistamines, and corticosteroids 
depending on the severity of their previous sting reaction. The only treatment to prevent further systemic sting reactions is venom immunotherapy [38].

AIT requires repeated contact between patient and physician/medical assistant/nurse over a longer period of time (3 to 5 years). In subcutaneous immunotherapy (SCIT), injections are given daily, weekly (up-dosing phase), or every 4-6 weeks (maintenance phase).

Most AIT products authorized for use in Europe indicate that AIT should be temporarily discontinued in case of infection; the same principle will apply to the COVID-19 pandemic. In patients on subcutaneous or sublingual (SLIT) AIT, who are diagnosed with COVID-19, those suspected of SARSCoV-2 infection, or symptomatic patients with a positive contact to SARS-CoV-2 individuals, the AIT should be interrupted until the patient has recovered. In patients not infected or who have recovered from the infection, AIT could be continued. These recommendations are conditional and could change as clinical data evolve $[5,39]$.

It is especially important to continue AIT in patients with life-threatening conditions like in venom allergy. If venom AIT was stopped due to SARS-CoV-2 infection, it is unclear when it should be re-initiated because data from convalescent patients is scarce [39].

It could start of AIT in allergic patients without known SARS-CoV-2 exposure who do not show COVID-19 symptoms, but whose current SARS-CoV-2 infection and immune status are unknown, requires a thorough examination for signs of SARS-CoV-2 infection during the indication for AIT, and the same examination should be repeated at the start of AIT.

An important consideration is SLIT, because it offers the possibility of taking the allergen preparation at home without further medical supervision after the first dose had been given in the allergy department. This avoids repeated visits to the allergy department, which is associated with an infection risk. On the other hand, the need to discontinue AIT can better be assessed by a physician, as patients may not be able to correctly correlate their symptoms to a potential COVID-19 infection. Subclinical/oligosymptomatic courses represent a particular challenge for patients on self-medication. Thus, patients taking SLIT AIT at home should be advised to contact their physician before applying the next dose if signs of infection occur.

When using SCIT preparations, the injection intervals can be prolonged in patients who have recovered from COVID-19 or in whom an adequate SARS-CoV-2 antibody reaction is detected after (possibly asymptomatic) disease can start or continue AIT as planned [39].

General recommendations for AIT administration during COVID-19 outbreak [39]:

1. The staff and all persons entering a practice/allergy department should take suitable measures for hand hygiene using soap and water or an alcohol-based hand disinfectant with an antiviral effect.

2. Based on a case-by-case risk assessment, the use of PPE should be considered in the AIT setting.

3. Only one person should accompany children and adolescents who must also apply all hygienic measures.

4. In each allergy center, one person (senior consultant, nurse, medical assistant) should lead the COVID-19 preventive measures and implement the corresponding guidelines for infrastructure and control measures.

5. Signs indicating the main symptoms suggestive of a COVID-19 infection (fever, cough, dyspnea, etc.) should be posted at all entrance doors, and visitors showing one of these symptoms should be advised not to enter the AIT practice/allergy department.

\section{Biological monoclonal antibodies}

A significant number of the allergic patients are on treatment with biologicals, and physicians face the challenge to provide optimal care during the pandemic. Uncertainty of the potential risks for these patients is related to the fact that the exact sequence of immunological events during SARS-CoV-2 is not known. Severe COVID-19 patients may experience a "cytokine storm" and associated organ damage characterized by an exaggerated release of pro-inflammatory type 1 and type 3 cytokines. These inflammatory responses are potentially counteracted by anti-inflammatory cytokines and type 2 responses.

The immune response after SARS-CoV-2 infection is so far only insufficiently understood and could be altered in a favorable or unfavorable way by therapy with monoclonal antibodies. There is currently no evidence for an increased risk of a severe COVID-19 course in allergic patients. The large Spanish sample (545 patients) report found that patients with severe asthma requiring a biologic treatment do not have an increased risk of COVID-19 infection or greater disease severity and mortality [40].

Many patients are under ongoing therapy with biologicals that block IgE antibodies or the interleukins IL-4, IL-5, and IL-13, which are relevant in their receptors or type 2 inflammation [41].

Omalizumab has been approved for the treatment of severe AA in adults and in children older than 6 years with proven sensitization against a perennial airborne allergen and reduced lung function. Another indication is antihistamine-resistant chronic spontaneous urticaria in adults and in adolescents older than 12 years. Mepolizumab, benralizumab, and reslizumab are IL-5 or IL-5 receptor blockers, available for adults and, partially, also for children. Dupilumab has been approved for the treatment of (a) atopic dermatitis in adolescents older than 12 years, (b) severe type-2-dominant asthma 
in adults, and (c) chronic rhinosinusitis with nasal polyps (CRSwNP) in adults [41].

According to the current state of knowledge, biological therapy for the indications discussed here can be continued in mild to moderate cases of SARS-CoV2 infection/COVID19 disease, if an individual consideration of risks and benefits supports this decision. The risks and benefits have to be assessed by a specialist, and it is recommended to inform the patient about the fact that only limited data are available.

In severe courses of COVID-19, prolongation of the dosing interval or treatment interruption should be considered. When doing so, the risk of the potential requirement of treatment with systemic glucocorticoids should also be taken into account $[42,43]$.

If hospitalization due to the exacerbation of asthma or type2-associated diseases becomes necessary, current guidelines on diagnosis and treatment must be followed. Sinus surgery for CRSwNP should, if possible, be delayed in patients with suspected or confirmed COVID-19 disease.

First data from Italy on dupilumab-treated non-infected in high epidemic areas, and current evidence from dupilumab trials, suggest no negative effect of dupilumab regarding viral infections [44] with reports on a reduced number of herpes simplex superinfections and less bacterial superinfections [45].

Anti-IgE treatment with omalizumab (or other biologics indicated for AA) should be continued in non-infected patients. Self-administration devices at home, whenever this option is available, are preferred, to minimize face-to-face contact in the clinic. In infected patients, omalizumab administration should be delayed until complete clinical recovery and viral clearance is achieved [46, 47].

The current EAACI statement on the usage of biologicals in the context of COVID-19 advices no change of therapy in non-infected individuals and to withhold/delay the application of biologicals for a minimum of 2 weeks or the resolution of the disease in case of SARS-CoV-2 infection [46]. This is based on expert opinion in the light of missing data and may be adapted if more information becomes available.

General recommendations for biological therapy during COVID-19 outbreak:

1. Telehealth follow-up should be considered in suitable patients when technical and medical requirements allow for it.

2. Comprehensive patient training with regard to documentation of the disease activity and, where applicable, to selfadministration of the medication is desirable. This is facilitated by the partial availability of user-friendly pen systems for self-application.

3. In countries with low infection numbers and a consequent relaxation of COVID-19-associated restrictions, there is no contraindication for starting biological therapy in patients without evidence of a current SARS-CoV-2 infection.

\section{Conclusions}

The COVID-19 pandemic has presented many challenges to the practicing allergist. Until the outbreaks are controlled, an effective vaccine and their consequent herd effect, and more effective treatments emerge, it is important to understand the scientific rationale that underlies pandemic mitigation strategies. Social distancing, hand disinfection, adaptations of consultation for patients, and adequate PPE for health care workers deserve further attention and promotion. It is highly recommended to prioritize $\mathrm{AD}$ patients and choose case-bycase those that might have a face-to-face consultation or treatment. Telehealth during COVID-19 times for AD patients must be encouraged whenever is possible, and telemedicine platform can provide a reliable service in patient care. Concerning the behavior and severity of COVID-19 infection in patients with $\mathrm{AD}$, and the course of $\mathrm{AD}$ in COVID-19 infection, the evidence is inconclusive and controversial. Further research is needed to clarify the relationship between them.

\section{Declarations}

Ethics Approval and Consent to Participate This article does not contain any studies with human or animal subjects performed by any of the authors.

Conflict of Interest The authors declare no competing interests.

\section{References}

Papers of particular interest, published recently, have been highlighted as:

- Of importance

• Of major importance

1. Hosoki K, Chakraborty A, Sur S. Molecular mechanisms and epidemiology of COVID-19 from an allergist's perspective. J Allergy Clin Immunol. 2020;146(2):285-99.

2. Chhiba KD, Patel GB, Vu THT, Chen MM, Guo A, Kudlaty E, et al. Prevalence and characterization of asthma in hospitalized and non-hospitalized patients with COVID-19. J Allergy Clin Immunol. 2020;146(2):307-14.e4.

3. Shaker MS, Oppenheimer J, Grayson M, Stukus D, Hartog N, Hsieh EWY, et al. COVID-19: pandemic contingency planning for the allergy and immunology clinic. J Allergy Clin Immunol Pract. 2020;8(5):1477-88.e5.

4. Olaguibel JM, Alobid I, Alvarez Puebla M, Crespo-Lessmann A, Domínguez Ortega J, García-Rio F, et al. Upper and lower airways functional examination in asthma and respiratory allergic diseases. Considerations in the SARS-CoV-2 post-pandemic situation. J Investig Allergol Clin Immunol. 2020. https://doi.org/10.18176/ jiaci.0625 COMMENT: this review shows the different examination techniques available on the assessment of patients suffering from respiratory allergy, asthma and associated diseases in the pandemic momentum. 
5.• Pfaar OKL, Klimek L, Jutel M, Akdis CA, Bousquet J, Breiteneder $\mathrm{H}$, et al. COVID-19 pandemic: practical considerations on the organization of an allergy clinic an EAACI/ARIA position paper. Allergy. 2020. https://doi.org/10.1111/all.14453 COMMENT: this consensus document offers a prioritization rational to help guide decision making when such situations arise and an allergist/immunologist is forced to reduce services or makes the de- cision on his or her own to do so.

6. Skoner DP. Allergic rhinitis: definition, epidemiology, pathophysiology, detection, and diagnosis. J Allergy Clin Immunol. 2001;108(1 Suppl):S2-8.

7. Brożek JL, Bousquet J, Agache I, Agarwal A, Bachert C, BosnicAnticevich S, et al. Allergic rhinitis and its impact on asthma (ARIA) guidelines-2016 revision. J Allergy Clin Immunol. 2017;140(4):950-8.

8. Mullol J, Del Cuvillo A, Lockey RF. Rhinitis phenotypes. J Allergy Clin Immunol Pract. 2020;8(5):1492-503.

9. Izquierdo-Dominguez A, Jauregui I, Del Cuvillo A, Montoro J, Davila I, Sastre J, et al. Allergy rhinitis: similarities and differences between children and adults. Rhinology. 2017;55(4):326-31.

10. Bousquet J, Khaltaev N, Cruz AA, Denburg J, Fokkens WJ, Togias A, et al. Allergic rhinitis and its impact on asthma (ARIA) 2008 update (in collaboration with the World Health Organization, GA(2)LEN and AllerGen). Allergy. 2008;63(Suppl 86):8-160.

11. Platts-Mills TA. Allergen avoidance. J Allergy Clin Immunol. 2004;113(3):388-91.

12. Bousquet J, Schünemann HJ, Togias A, Bachert C, Erhola M, Hellings PW, et al. Next-generation allergic rhinitis and its impact on asthma (ARIA) guidelines for allergic rhinitis based on Grading of Recommendations Assessment, Development and Evaluation (GRADE) and real-world evidence. J Allergy Clin Immunol. 2020;145(1):70-80.e3.

13. Yang JM, Koh HY, Moon SY, Yoo IK, Ha EK, You S, et al. Allergic disorders and susceptibility to and severity of COVID19: a nationwide cohort study. J Allergy Clin Immunol. 2020;146(4):790-8.

14. Scadding GK, Hellings PW, Bachert C, Bjermer L, Diamant Z, Gevaert $\mathrm{P}$, et al. Allergic respiratory disease care in the COVID19 era: A EUFOREA statement. World Allergy Organ J. 2020;13(5):100124.

15. Bousquet J, Akdis C, Jutel M, Bachert C, Klimek L, Agache I, et al. Intranasal corticosteroids in allergic rhinitis in COVID-19 infected patients: An ARIA-EAACI statement. Allergy. 2020;75:2440-4. https://doi.org/10.1111/all.14302.

16. Klimek L, Jutel M, Akdis C, Bousquet J, Akdis M, Bachert C, et al. Handling of allergen immunotherapy in the COVID-19 pandemic: an ARIA-EAACI statement. Allergy. 2020;75(7):1546-54.

17. Hartmann-Boyce J, Gunnell J, Drake J, Otunla A, Suklan J, Schofield E, et al. Asthma and COVID-19: review of evidence on risks and management considerations. BMJ Evid Based Med. 2020. https://doi.org/10.1136/bmjebm-2020-111506 COMMENT: In this review, the authors set out to review existing publications, preprints and national guidelines to answer the risk and management in asthmatic patients during the COVID-19 pandemic.

18. Global Initiative for Asthma. Global strategy for asthma management and prevention, 2020. Available from: www.ginaasthma.org. Accesed 11 Nov 2020.

19. Guía Española para el Manejo del Asma (GEMA5.0). Global Strategy for Asthma Management, 2019. Available from: www. gemasma.com

20. Abrams EM, 't Jong GW, Yang CL. Asthma and COVID-19. CMAJ. 2020;192(20):E551

21. Izquierdo-Domínguez $\mathrm{A}$, Rojas-Lechuga MJ, Chiesa-Estomba $\mathrm{C}$, Calvo-Henríquez C, Ninchritz-Becerra E, Soriano-Reixach M, et al. Smell and taste dysfunction in COVID-19 is associated with younger age in ambulatory settings: a multicenter cross-sectional study. J Investig Allergol Clin Immunol. 2020;30(5):346-57.

22. Coronavirus Disease 2019 (COVID-19)-People with Certain Medical Conditions. https://www.cdc.gov/coronavirus/2019-ncov/ need-extra-precautions/people-with-medicalconditions.html? CDC_AA_refVal=https\%3 A \% 2F\%2Fwww.cdc.gov\% 2 Fcoronavirus $\% 2$ F2019-ncov $\% 2$ Fneed-extra-precautions $\%$ 2Fgroups-at-higher-risk.html. Last Updated Nov. 2, 2020. Content source: National Center for Immunization and Respiratory Diseases (NCIRD), Division of Viral Diseases. Accesed 11 Nov 2020

23. Williamson EJ, Walker AJ, Bhaskaran K, Bacon S, Bates C, Morton CE, et al. Factors associated with COVID-19-related death using OpenSAFELY. Nature. 2020;584(7821):430-6.

24. Bousquet J, Jutel M, Akdis CA, Klimek L, Pfaar O, Nadeau KC, et al. ARIA-EAACI statement on asthma and COVID-19 (June 2, 2020). Allergy. 2020:2020. https://doi.org/10.1111/all.14471.

25. Muraro A, Werfel T, Hoffmann-Sommergruber K, Roberts G, Beyer K, Bindslev-Jensen C, et al. EAACI food allergy and anaphylaxis guidelines: diagnosis and management of food allergy. Allergy. 2014;69(8):1008-25.

26. Protudjer JLP, Golding M, Salisbury MR, Abrams EM, Roos LE. High anxiety andhealth-related quality of life in families with children with food allergy during coronavirus disease 2019. Ann Allergy Asthma Immunol. 2021:2020. https://doi.org/10.1016/j. anai.2020.09.010

27. Mack DP, Chan ES, Shaker M, Abrams EM, Wang J, Fleischer DM, et al. Novel approaches to food allergy management during COVID-19 inspire long-term change. J Allergy Clin Immunol Pract. 2020;8(9):2851-7. https://doi.org/10.1016/j.jaip.2020.07. 020 .

28. Brockow K, Ardern-Jones MR, Mockenhaupt M, Aberer W, Barbaud A, Caubet JC, et al. EAACI position paper on how to classify cutaneous manifestations of drug hypersensitivity. Allergy. 2019;74(1):14-27.

29. Patil SS, Sun L, Fox CJ, Anthony KE, Anzalone FA, Fisher PM, et al. Multiple drug allergies: recommendations for perioperative management. Best Pract Res Clin Anaesthesiol. 2020;34(2):32544.

30. Gelincik A, Brockow K, Celik GE, Doña I, Mayorga C, Romano A, et al. Diagnosis and management of the drug hypersensitivity reactions in Coronavirus disease 19: an EAACI position paper. Allergy. 2020;75(11):2775-93.

31. Dordal Culla MT, Herrera-Lasso Regás V, Martí Garrido J, Rodríguez Cumplido D, Vázquez-Revuelta P, Lleonart Bellfill R. Treating COVID-19: review of drug hypersensitivity reactions. J Investig Allergol Clin Immunol. 2020. https://doi.org/10.18176/ jiaci.0588 COMMENT: the document summarizes current knowledge regarding mainly the immunological adverse drug reactions related to the drugs used for COVID-19, in order to address their management in a comprehensive manner for allergology study.

32. Estébanez A, Pérez-Santiago L, Silva E, Guillen-Climent S, GarcíaVázquez A, Ramón MD. Cutaneous manifestations in COVID-19: a new contribution. J Eur Acad Dermatol Venereol. 2020;34(6): e250-1.

33. Suchonwanit P, Leerunyakul K, Kositkuljorn C. Cutaneous manifestations in COVID-19: lessons learned from current evidence. J Am Acad Dermatol. 2020;83(1):e57-60.

34. Wollenberg A, Flohr C, Simon D, Cork MJ, Thyssen JP, Bieber T, et al. European Task Force on Atopic Dermatitis statement on severe acute respiratory syndrome coronavirus 2 (SARS-Cov-2) infection and atopic dermatitis. J Eur Acad Dermatol Venereol. 2020;34(6):e241-2.

35. Ferrucci S, Romagnuolo M, Angileri L, Berti E, Tavecchio S. Safety of dupilumab in severe atopic dermatitis and infection of 
Covid-19: two case reports. J Eur Acad Dermatol Venereol. 2020;34(7):e303-4.

36. Yan Y, Chen H, Chen L, Cheng B, Diao P, Dong L, et al. Consensus of Chinese experts on protection of skin and mucous membrane barrier for health-care workers fighting against coronavirus disease 2019. Dermatol Ther. 2020;33(4):e13310.

37. Bousquet J, Pfaar O, Togias A, Schünemann HJ, Ansotegui I, Papadopoulos NG, Tsiligianni I, Agache I, Anto JM, Bachert C, Bedbrook A, Bergmann KC, Bosnic-Anticevich S, Bosse I, Brozek J, Calderon MA, Canonica GW, Caraballo L, Cardona V, Casale T, Cecchi L, Chu D, Costa E, Cruz AA, Czarlewski W, Durham SR, du Toit G, Dykewicz M, Ebisawa M, Fauquert JL, FernandezRivas M, Fokkens WJ, Fonseca J, Fontaine JF, Gerth van Wijk $\mathrm{R}$, Haahtela T, Halken S, Hellings PW, Ierodiakonou D, Iinuma T, Ivancevich JC, Jacobsen L, Jutel M, Kaidashev I, Khaitov M, Kalayci O, Kleine Tebbe J, Klimek L, Kowalski ML, Kuna P, Kvedariene V, la Grutta S, Larenas-Linemann D, Lau S, Laune D, le L, Lodrup Carlsen K, Lourenço O, Malling HJ, Marien G, Menditto E, Mercier G, Mullol J, Muraro A, O’Hehir R, Okamoto Y, Pajno GB, Park HS, Panzner P, Passalacqua G, Pham-Thi N, Roberts G, Pawankar R, Rolland C, Rosario N, Ryan D, Samolinski B, Sanchez-Borges M, Scadding G, Shamji MH, Sheikh A, Sturm GJ, Todo Bom A, Toppila-Salmi S, Valentin-Rostan M, Valiulis A, Valovirta E, Ventura MT, Wahn U, Walker S, Wallace D, Waserman S, Yorgancioglu A, Zuberbier T, the ARIA Working Group ARIA Working Group. 2019 ARIA Care pathways for allergen immunotherapy. Allergy. 2019;74(11):2087-2102.

38. Sturm GJ, Varga EM, Roberts G, Mosbech H, Bilò MB, Akdis CA, et al. EAACI guidelines on allergen immunotherapy: hymenoptera venom allergy. Allergy. 2018;73(4):744-64.

39. Klimek L, Pfaar O, Worm M, Bergmann KC, Bieber T, Buhl R, et al. Allergen immunotherapy in the current COVID-19 pandemic: a position paper of AeDA, ARIA, EAACI, DGAKI and GPA: position paper of the German ARIA Group in cooperation with the Austrian ARIA Group, the Swiss ARIA, German Society for Applied Allergology (AEDA), German Society for Allergology and Clinical Immunology (DGAKI), Society for Pediatric Allergology (GPA) in cooperation with AG Clinical Immunology, Allergology and Environmental Medicine of the DGHNO-KHC, and the European Academy of Allergy and Clinical Immunology (EAACI). Allergol Select. 2020;4:44-52.

40. Rial MJ, Valverde M, Del Pozo V, González-Barcala FJ, MartínezRivera C, Muñoz X, et al. Clinical characteristics in 545 patients with severe asthma on biological treatment during the COVID-19 outbreak. J Allergy Clin Immunol Pract. 2020;9:487-489.e1. https://doi.org/10.1016/j.jaip.2020.09.050.

41. Klimek L, Pfaar O, Worm M, Eiwegger T, Hagemann J, Ollert M, et al. Use of biologicals in allergic and type- 2 inflammatory diseases during the current COVID-19 pandemic: position paper of Ärzteverband Deutscher Allergologen (AeDA) A, Deutsche Gesellschaft für Allergologie und Klinische Immunologie (DGAKI) B, Gesellschaft für Pädiatrische Allergologie und Umweltmedizin (GPA)C, Österreichische Gesellschaft für Allergologie und Immunologie (ÖGAI) D, Luxemburgische Gesellschaft für Allergologie und Immunologie (LGAI) E, Österreichische Gesellschaft für Pneumologie (ÖGP) F in cooperation with the German, Austrian, and Swiss ARIA groupsG, and the European Academy of Allergy and Clinical Immunology (EAACI) H. Allergol Select. 2020;7(4):53-68.

42. Pajno GB, Fernandez-Rivas M, Arasi S, Roberts G, Akdis CA, Alvaro-Lozano $\mathrm{M}$, et al. EAACI guidelines on allergen immunotherapy: IgE-mediated food allergy. Allergy. 2018;73:799-815.

43. Roberts G, Pfaar O, Akdis CA, Ansotegui IJ, Durham SR, Gerth van Wijk R, et al. EAACI guidelines on allergen immunotherapy: allergic rhinoconjunctivitis. Allergy. 2018;73:765-98.

44. Carugno A, Raponi F, Locatelli AG, Vezzoli P, Gambini DM, Di Mercurio $\mathrm{M}$, et al. No evidence of increased risk for coronavirus disease 2019 (COVID-19) in patients treated with dupilumab for atopic dermatitis in a high-epidemic area - Bergamo, Lombardy, Italy. J Eur Acad Dermatol Venereol. 2020. https://doi.org/10. $1111 /$ jdv.16552.

45. Simpson EL, Paller AS, Siegfried EC, Boguniewicz M, Sher L, Gooderham MJ, et al. Efficacy and safety of dupilumab in adolescents with uncontrolled moderate to severe atopic dermatitis: a phase 3 randomized clinical trial. JAMA Dermatol. 2020;156(1): $44-56$.

46. Vultaggio A, Agache I, Akdis CA, Akdis M, Bavbek S, Bossios A, et al. Considerations on biologicals for patients with allergic disease in times of the COVID-19 pandemic: an EAACI statement. Allergy. 2020;75(11):2764-74.

47. Morais-Almeida M, Aguiar R, Martin B, Ansotegui IJ, Ebisawa M, Arruda LK, et al. COVID-19, asthma, and biological therapies: what we need to know. World Allergy Organ J. 2020;13(5): 100126.

Publisher's Note Springer Nature remains neutral with regard to jurisdictional claims in published maps and institutional affiliations. 